\title{
Monkeys and Rats Are Not Susceptible to Ferret Hepatitis E Virus Infection
}

\author{
Tian-Cheng $\mathrm{Li}^{\mathrm{a}}$ Sayaka Yoshizaki ${ }^{\mathrm{a}}$ Yasushi Ami ${ }^{\mathrm{b}}$ Yuriko Suzaki ${ }^{\mathrm{b}}$ \\ Tingting Yang ${ }^{d}$ Naokazu Takedac Wakita Takaji ${ }^{\mathrm{a}}$ \\ aDepartment of Virology II, and b Division of Experimental Animal Research, National Institute of Infectious Diseases, \\ Tokyo, and ' Research Institute for Microbial Diseases, Osaka University, Osaka, Japan; ${ }^{d}$ Department of Clinical \\ Laboratory, Affiliated Hospital of Qingdao University Medical College, Qingdao, China
}

\section{Key Words}

Ferret hepatitis E virus · Laboratory rat · Nude rat · Monkey

\begin{abstract}
Ferret hepatitis E virus (HEV), a novel hepatitis E-like virus, has been identified in ferrets in the Netherlands, Japan, and the US. To determine whether ferret HEV transmits to other animals, we inoculated laboratory rats (Wistar), nude rats (Long-Evans-rnu/rnu), and cynomolgus monkeys with ferret HEV (F4351) by intravenous injection. None of the animals demonstrated a positive sign for virus replication, indicating that rats and monkeys are not susceptible to ferret HEV.
\end{abstract}

๑) 2015 S. Karger AG, Basel

Hepatitis E virus (HEV), a small round nonenveloped single-strand-positive RNA virus, is the sole member of the genus Hepevirus in the family Hepeviridae [1]. Hepevirus includes four genotypes of HEV that have been isolated from humans (G1, G2, G3, and G4 HEV) and cause acute hepatitis E [2]. Swine, wild boars, deer, and mongooses are the reservoirs for G3 and G4 HEV, and these HEVs can be transmitted from these animals to humans, suggesting that hepatitis $\mathrm{E}$ is a zoonotic disease [3]. In ad- dition to G1-G4 HEV, numerous HEV s or HEV-like viruses have been isolated from rabbits, bats, birds, wild rats, red foxes, minks, moose, and ferrets [4-10], and Hepevirus may include at least four species of HEV: G1G4/rabbit/unclassified wild boar HEV, avian HEV, bat $\mathrm{HEV}$, and rat/ferret HEV [11].

The genome structure of the ferret HEV is similar to that of the other HEVs, containing three open reading frames (ORFs): ORF1 encodes a nonstructural protein, ORF2 encodes a capsid protein, and ORF3 encodes a phosphoprotein with multifunction. The serotype of ferret HEV is different from that of G1-G4 HEV [12]. After ferret HEV was first identified in ferrets in the Netherlands, it was also detected in laboratory ferrets in the US and in imported ferrets in Japan [12]. Expression of ferret HEV ORF2 allowed the partial capsid protein to assemble into virus-like particles, and an enzyme-linked immunosorbent assay (ELISA) for the detection of anti-ferret HEV IgG and IgM antibodies using the virus-like particles as the antigen has been established [12]. However, the pathogenicity and epidemiology of ferret HEV are largely unknown.

Because ferrets have been used not only as a small animal model for virus infections but also kept as pets, studies to determine whether ferret HEV transmits to humans

\section{KARGER 125}

(c) 2015 S. Karger AG, Basel

0300-5526/15/0583-0139\$39.50/0

E-Mail karger@karger.com

www.karger.com/int
Tian-Cheng Li

Department of Virology II, National Institute of Infectious Diseases

4-7-1 Gakuen, Musashi-murayama

Tokyo 208-0011 (Japan)

E-Mail litc@nih.go.jp 
Fig. 1. Kinetics of biochemical, serological, and virological markers after inoculation. Laboratory ferrets were inoculated with ferret HEV F4351. Ferret HEV RNA in the stool and serum was monitored by RTPCR: +, positive; -, negative. Anti-ferret HEV IgG $(O)$ and $\operatorname{IgM}(\triangle)$ antibodies were detected by ELISA, and the animals' ALT ( $\square$ ) levels were monitored.
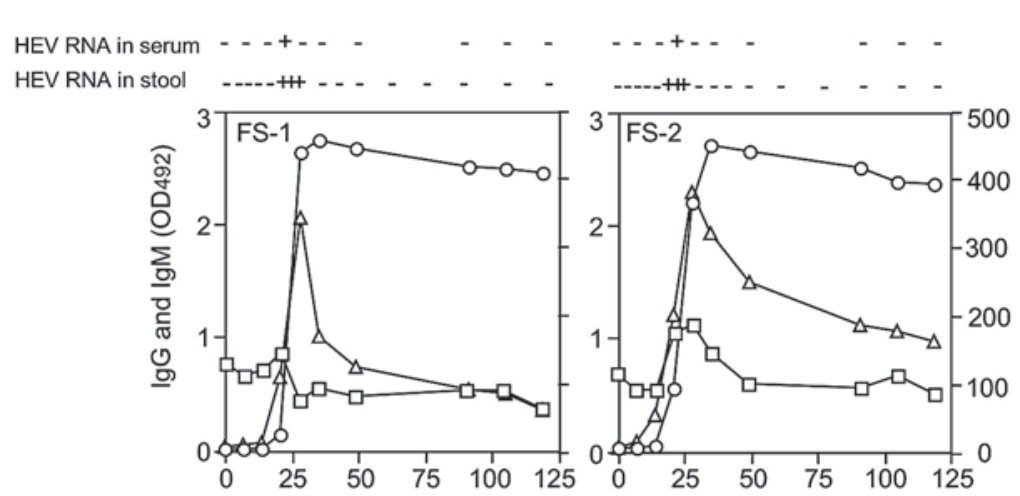

$\stackrel{\unrhd}{\Xi}$

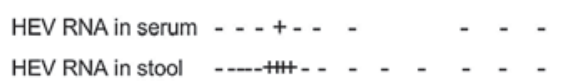

- - - . - - -

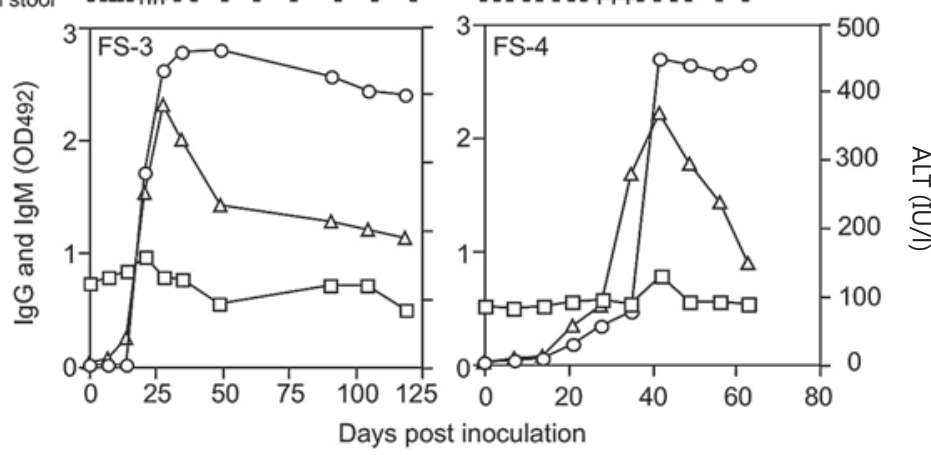

or other animals are urgently needed. In the present study, we inoculated SPF rats, nude rats, and cynomolgus monkeys with ferret HEV, and we monitored the virus replication to determine the susceptibility of rats and monkeys to ferret HEV infection.

We used the ferret HEV strain F4351 (GenBank accession No. AB890001) derived from stool specimens from a laboratory ferret (Macaca fascicularis furo) for the infection experiments [13]. The stool specimen containing F4351 was diluted with $10-\mathrm{mM}$ phosphate-buffered saline to prepare a $10 \%$ suspension, shaken at $4^{\circ}$ for $1 \mathrm{~h}$, and clarified by centrifugation at $10,000 \mathrm{~g}$ for $30 \mathrm{~min}$. The supernatant was passed through a $0.45-\mu \mathrm{m}$ membrane filter (Millipore, Bedford, Mass., USA) and stored at $-80^{\circ}$ until use. The RNA copy number of F4351 was detected as $2 \times$ $10^{5}$ copies/ml by real-time reverse transcription-polymerase chain reaction (RT-PCR).

Three 15-week-old SPF rats (Wistar, Japan SLC, Hamamatsu, Japan), three 5-week-old nude rats (LongEvans-rnu/rnu, Japan SLC), three 11-year-old cynomolgus monkeys, three 22-week-old ferrets, and one 2-yearold ferret (Japan SLC) were used. All of the animals were shown to be negative for ferret and rat HEV RNA and anti-G1, G3, G4, ferret, and rat HEV antibodies by a nested broad-spectrum RT-PCR and ELISA, respectively.

To examine the susceptibility of the laboratory animals to ferret HEV, the SPF and nude rats were intravenously inoculated with $0.5 \mathrm{ml}$ of F4351 through the tail vein, whereas the monkeys were intravenously inoculated with $1.0 \mathrm{ml}$ of F4351 through a femoral vein. Three ferrets (FS-1, FS-2, and FS-3) were orally inoculated with $2.0 \mathrm{ml}$ of F 4351 mixed with $10 \mathrm{ml}$ of milk for 3 consecutive days, and one ferret (2-year-old, FS-4) was inoculated with 1.0 $\mathrm{ml}$ of F4351 through the cranial vena cava.

The serum samples were collected weekly to examine ferret HEV RNA as well as ferret HEV-specific IgG and IgM antibodies. Sera were also used to determine the animal's alanine aminotransferase (ALT) levels. Stool samples were collected every 3 days after inoculation to detect HEV RNA. The animals were monitored for 3 months after inoculation, with the exception of ferret FS-4, which was monitored until day 63 after inoculation. All of the animal experiments were reviewed and carried out according to the 'Guidelines for Animal Experiments Per- 
formed at the National Institute of Infectious Diseases' under codes 513005 and 614006. The animals were individually housed in BSL-2 facilities.

The extraction of RNA was carried out using the MagNA Pure LC Total Nucleic Acid Isolation Kit (Roche Applied Science, Mannheim, Germany) according to the manufacturer's recommendations. Reverse transcription was performed with a high-capacity cDNA reverse transcription kit (ABI Applied Biosystems, Carlsbad, Calif., USA). A nested broad-spectrum RT-PCR targeting a portion of the ORF1 genome was performed as described with a slight modification [14]. The nested PCR was carried out with a forward primer, HEV-cs, and an internal reverse primer, HEV-casn. The ferret HEV RNA titer was detected by one-step quantitative RT-PCR with forward (5'-TGAGTGGTGTTTTTATGGCGA-3', nt 4006-4026) and reverse (5'-CAAACTCAGAAAAATCATTCTCAAAGAC- $3^{\prime}$, nt 4082-4109) primers, and a probe $\left(5^{\prime}\right.$ 6FAM-GCCATGCCGCGGTTTGAGGCGGCTGTTAMRA-3', nt 4034-4059). Anti-rat, monkey, and ferret HEV IgG and IgM antibodies were detected by ELISA as described $[12,15]$. Horseradish peroxidase (HRP)-conjugated goat anti-rat IgG $(\mathrm{H}+\mathrm{L}$; Zymed Laboratories, San Francisco, Calif., USA), IgM (Jackson Immunoresearch Laboratories, West Grove, Pa., USA), HRP-conjugated goat anti-human IgG (Cappel, West Chester, Pa., USA), IgM (Cappel), HRP-conjugated goat anti-ferret IgG $(\mathrm{H}+\mathrm{L}$; KPL, Gaithersburg, Md., USA), and IgM (Rockland Antibodies and Assays, Gilbertsville, Pa., USA) were used to detect rat, monkey, and ferret antibodies, respectively. Serum ALT levels were monitored weekly using the Fuji Dri-Chem Slide GPT/ALT-PIII kit (Fujifilm, Saitama, Japan).

To examine the infectivity of ferret HEV F4351, we orally inoculated three ferrets with $2 \mathrm{ml}$ of $10 \%$ stool suspension. As shown in figure 1, ferret HEV RNA was detected in stools of all three ferrets from 19 to 26 days after inoculation. However, the RNA titer was measurable only in ferret FS-2 at 18, 21, and 24 days after inoculation with $2.47 \times 10^{4}, 2.37 \times 10^{7}$, and $6.48 \times 10^{6}$ copies/g, respectively. Anti-ferret HEV IgG and IgM antibodies were detected in the sera at 3 weeks after inoculation in all three ferrets. The IgM antibody titers reached their peak at 4 weeks after inoculation and then gradually decreased, whereas the IgG antibody titers reached their peak at 4 weeks and remained at high levels until the end of the experiment. Ferret HEV RNA in the serum samples was detected at 3 weeks after inoculation in these ferrets. During the ferret HEV RNA-positive period, a slight elevation of the ALT levels was observed. These results indicate that
Table 1. Animals and detection of ferret HEV RNA, anti-ferret HEV antibodies, and ALT levels after inoculation

\begin{tabular}{|c|c|c|c|c|c|}
\hline Animals & Sex & $\begin{array}{l}\text { Virus inoculum } \\
\text { (in genome } \\
\text { equivalent copies) }\end{array}$ & $\begin{array}{l}\text { Ferret } \\
\text { HEV } \\
\text { RNA }\end{array}$ & $\begin{array}{l}\text { IgG } \\
\text { and } \\
\text { IgM }\end{array}$ & $\begin{array}{l}\text { ALT } \\
(\mathrm{IU} / \mathrm{l})^{\mathrm{a}}\end{array}$ \\
\hline Rat-1 (Wistar) & M & $10^{5}$ & Neg & Neg & $<40$ \\
\hline Rat-2 (Wistar) & M & $10^{5}$ & Neg & Neg & $<40$ \\
\hline Rat-3 (Wistar) & M & $10^{5}$ & $\mathrm{Neg}$ & Neg & $<40$ \\
\hline $\begin{array}{l}\text { Long-Evans-rnu/ } \\
\text { rnu-1 }\end{array}$ & $\mathrm{F}$ & $10^{5}$ & Neg & Neg & $<40$ \\
\hline $\begin{array}{l}\text { Long-Evans-rnu/ } \\
\text { rnu-2 }\end{array}$ & F & $10^{5}$ & Neg & Neg & $<40$ \\
\hline $\begin{array}{l}\text { Long-Evans-rnu/ } \\
\text { rnu-3 }\end{array}$ & F & $10^{5}$ & Neg & Neg & $<40$ \\
\hline Monkey 4824 & $\mathrm{~F}$ & $2 \times 10^{5}$ & Neg & Neg & $<60$ \\
\hline Monkey 4826 & M & $2 \times 10^{5}$ & Neg & Neg & $<60$ \\
\hline Monkey 4830 & M & $2 \times 10^{5}$ & Neg & Neg & $<60$ \\
\hline Ferret-FS4 & M & $2 \times 10^{5}$ & Pos & Pos & $<150$ \\
\hline
\end{tabular}

$\mathrm{Neg}=$ Negative: during the whole period of the experiment the sera were negative for ferret HEV RNA, or IgG and IgM; Pos = positive for ferret HEV RNA, or IgG and IgM. The positive period is shown in fig. $1{ }^{\text {a }}$ The highest ALT level during the whole period of the experiment is shown.

ferret HEV F4351 is infectious and that ferret HEV was transmitted through the fecal-oral route. When ferret FS-4 was inoculated with F4351 through the cranial vena cava, ferret HEV RNA was detected in stools from 21 to 27 days after inoculation, and anti-ferret HEV IgG and IgM antibodies were detected in the sera at 3 weeks and reached their peak at 6 weeks after inoculation. A significant elevation of the ALT level was not observed. This result indicates that ferret $\mathrm{HEV}$ is also transmitted through intravenous inoculation.

All of the SPF rats, nude rats, and monkey serum samples collected from 1 to 13 weeks after inoculation were negative for ferret HEV RNA and anti-ferret HEV IgG and IgM antibodies. Elevated ALT levels were not observed in these serum samples (table 1). Consistent with the above results, ferret HEV RNA was not detected in the rat, nude rat, or monkey stool samples. These results indicate that ferret HEV is unable to infect laboratory rats, nude rats, or cynomolgus monkeys.

Two new proposals for an HEV taxonomy have been published. One proposal suggests that rat and ferret HEVs belong to the same species Orthohepevirus C [16], and the other suggests that these two HEVs are different species in the genus Rocahepevirus [17]. However, the nucleotide sequence analyses indicated that the ferret HEV genome 
shared $60.8-68.8 \%$ identity with rat HEVs, which is higher than those with G1-G4, rabbit, bird, and bat HEVs (46.6-55.2\%), and this is the reason why we selected rats to examine whether ferret HEV was transmissible to other animals. However, our findings even with nude rats did not support any evidence of ferret HEV replication. Here we selected a nonhuman primate (cynomolgus monkey) and inoculated three monkeys with ferret HEV; we found no evidence that cynomolgus monkeys were susceptible to ferret HEV. Our results demonstrated that rats and cynomolgus monkeys are not susceptible to ferret HEV.
However, it is necessary to clarify whether ferret HEV is transmissible to humans and causes a zoonotic disease, especially in the ferret feeders.

\section{Acknowledgements}

This study was supported in part by grants for Research on Emerging and Re-Emerging Infectious Diseases, Research on Hepatitis, and Research on Food Safety from the Ministry of Health, Labor, and Welfare, Japan.

\section{References}

1 Meng XJ, Anderson DA, Arankalle VA, Emerson SU, Harrison TJ, Jameel S, Okamoto H: Hepeviridae; in King AMQ, Adams MJ, Carstens EB, Lefkowitz EJ (eds): Virus Taxonomy: Classification and Nomenclature of Viruses. Ninth Report of the International Committee on Taxonomy of Viruses. London, Academic Press, 2012, pp 1021-1028.

- Emerson SU, Purcell RH: Hepatitis E virus. Rev Med Virol 2003;13:145-154.

3 Meng XJ: Hepatitis E virus: animal reservoirs and zoonotic risk. Vet Microbiol 2010;140: 256-265.

4 Zhao C, Ma Z, Harrison TJ, Feng R, Zhang C, Qiao Z, Fan J, Ma H, Li M, Song A, Wang Y: A novel genotype of hepatitis $\mathrm{E}$ virus prevalent among farmed rabbits in China. J Med Virol 2009;81:1371-1379.

5 Raj VS, Smits SL, Pas SD, Provacia LB, Moorman-Roest $\mathrm{H}$, Osterhaus AD, Haagmans BL: Novel hepatitis E virus in ferrets, the Netherlands. Emerg Infect Dis 2012;18:1369-1370.

6 Johne R, Heckel G, Plenge-Bönig A, Kindler E, Maresch C, Reetz J, Schielke A, Ulrich RG: Novel hepatitis E virus genotype in Norway rats, Germany. Emerg Infect Dis 2010;16: 1452-1455.

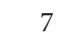

Drexler JF, Seelen A, Corman VM, Fumie Tateno A, Cottontail V, Melim Zerbinati R, Gloza-Rausch F, Klose SM, Adu-Sarkodie Y, Oppong SK, Kalko EK, Osterman A, Rasche A, Adam A, Müller MA, Ulrich RG, Leroy EM, Lukashev AN, Drosten C: Bats worldwide carry hepatitis E virus-related viruses that form a putative novel genus within the family Hepeviridae. J Virol 2012;86:91349147.

-8 Bodewes R, van der Giessen J, Haagmans BL, Osterhaus AD, Smits SL: Identification of multiple novel viruses, including a parvovirus and a hepevirus, in feces of red foxes. J Virol 2013;87:7758-7764.

-9 Krog JS, Breum SO, Jensen TH, Larsen LE: Hepatitis E virus variant in farmed mink, Denmark. Emerg Infect Dis 2013;19:20282030.

10 Jay Lin, Heléne Norder, Henrik Uhlhorn, Sándor Belák, Widén F: Novel hepatitis E like virus found in Swedish moose. J Gen Virol 2014;95:557-570.

11 Smith DB, Purdy MA, Simmonds P: Genetic variability and the classification of hepatitis $E$ virus. J Virol 2013;87:4161-4169.

12 Yang T, Kataoka M, Ami Y, Suzaki Y, Kishida N, Shirakura M, Imai M, Asanuma H, Takeda N, Wakita T, Li TC: Characterization of selfassembled virus-like particles of ferret hepatitis $\mathrm{E}$ virus generated by recombinant baculoviruses. J Gen Virol 2013;94:2647-2656.
13 Li TC, Yang T, Ami Y, Suzaki Y, Shirakura M, Kishida N, Asanuma H, Takeda N, Takaji W: Complete genome of hepatitis $\mathrm{E}$ virus from laboratory ferrets. Emerg Infect Dis 2014;20: 709-712.

14 Johne R, Plenge-Bonig A, Hess M, Ulrich RG, Reetz J, Schielke A: Detection of a novel hepatitis E-like virus in faeces of wild rats using a nested broad-spectrum RT-PCR. J Gen Virol 2010;91:750-758.

15 Li TC, Yoshimatsu K, Yasuda SP, Arikawa J, Koma T, Kataoka M, Ami Y, Suzaki Y, Mai le TQ, Hoa NT, Yamashiro T, Hasebe F, Takeda $\mathrm{N}$, Wakita T: Characterization of self-assembled virus-like particles of rat hepatitis $\mathrm{E}$ virus generated by recombinant baculoviruses. J Gen Virol 2011;92:2830-2837.

16 Smith DB, Simmonds P, Jameel S, Emerson SU, Harrison TJ, Meng XJ, Okamoto H, Van der Poel WH, Purdy MA: Consensus proposals for classification of the family Hepeviridae. J Gen Virol 2014;95:2223-2232.

17 Johne R, Dremsek P, Reetz J, Heckel G, Hess M, Ulrich RG: Hepeviridae: an expanding family of vertebrate viruses. Infect Genet Evol 2014;27C:212-229. 